\title{
REVISIONES
}

\section{Actividades de intervención educativa y de difusión del patrimonio organístico en entornos formales y no formales}

\author{
Educational intervention and dissemination of organistic heritage \\ in formal and non-formal environments \\ Miguel Díaz-Emparanza Almoguera, ${ }^{a}$ Juan Peruarena Arregui, ${ }^{a b}$ \\ Raquel Jiménez Pasalodos ${ }^{a c}$ \\ aniversidad de Valladolid \\ Telf.: (34) 983423596. Correo electrónico: mdiazemparanza@gmail.com \\ 'Telf.: (34) 983423596. Correo electrónico: jon.parregui@ gmail.com \\ cTelf.: (34) 983423596. Correo electrónico: rebiab@ hotmail.com
}

\begin{abstract}
RESUMEN
Desde hace más de doce años un equipo de investigadores de la Universidad de Valladolid ha venido desarrollando una constante actuación en torno al patrimonio musical organístico de Castilla y León, concretamente en su tipología barroca, cuyo volumen total de instrumentos supera los 250 ejemplares solo en las provincias de Valladolid y Palencia. A través de convenios suscritos entre la Universidad de Valladolid, las instituciones religiosas de ambas provincias y sus Diputaciones, se han elaborado múltiples actividades de tipo formativo, así como de difusión y protección del propio instrumento, mediante acciones vinculadas a visitas de carácter didáctico a Centros de Enseñanza enmarcadas en acciones formativas no formales pero dirigidas a entornos formales. Se incluyen, además, otras actividades complementarias destinadas a divulgar los bienes a través de su función y uso (liturgia como elemento de difusión patrimonial) y a integrarlos en redes de investigación de patrimonio histórico, todas ellas realizadas por alumnos de las titulaciones ofertadas por la Universidad de Valladolid. Se describen por tanto diversas propuestas de formación en patrimonio musical enfocadas a alumnado de distintas etapas educativas.
\end{abstract}

Palabras clave: órgano de tubos, patrimonio musical, patrimonio público, enseñanza del patrimonio histórico, conciencia histórica, acuerdo de colaboración.

ABSTRACT

For more than twelve years, an important activity around the organistic musical heritage of Castile and León has been developed by members of the University of Valladolid. Specific actions have been carried about some of the 250 baroque organs, located in the provinces of Valladolid and Palencia. Through successive partnership agreements signed between the University of Valladolid, religious institutions of both provinces, and their Provincial Councils, a reasonable number of activities have been developed to train musicians, as well as to preserve and disseminate these musical instruments. The activities comprise many didactic actions related to informal education in primary and secondary schools. Complementary activities are also implemented in order to disseminate historical heritage through its liturgical uses and functions, and to integrate it in historic heritage research networks. All activities are carried out by students of the several degrees in music offered by the University of Valladolid. This paper presents some proposals for the training in musical heritage of different kinds of students, according to the specific educational stages.

Keywords: pipe organ, musical heritage, public heritage, historic heritage education, historic awareness, partnership agreement. 


\section{ANTECEDENTES}

Debido a su rico patrimonio, las provincias de Valladolid y Palencia presentan, en el seno de la Comunidad de Castilla y León, una dilatada trayectoria alrededor de la estimación, reivindicación y valoración del patrimonio organístico de sus respectivos territorios, tal y como lo demuestra la secuencia de diversas iniciativas auspiciadas por individuos, asociaciones e instituciones desde mediados del pasado siglo. Un interés que se mantiene en la actualidad.

En el caso concreto de Valladolid, además de los estudios realizados por Esteban García Chico (1953, 1956), a finales de los años setenta del siglo XX surge un grupo de personas que se denominaban a sí mismas en la correspondencia conservada, "entusiastas del órgano y que sienten la preocupación de conocer y salvaguardar el patrimonio organístico vallisoletano, $[\mathrm{y}]$ que comienza a trabajar en un proyecto de catalogación de los órganos existentes en nuestra provincia" (Virgili, 2005). Este grupo de entusiastas, formado originalmente por Lucía Riaño, Federico Acitores y Jesús Ángel de la Lama, fue el germen de variadas actuaciones que han demostrado ser fundamentales para entender el momento actual de la recuperación de este patrimonio. Entre sus primeras acometidas se verificó la elaboración por Jesús Ángel de la Lama del catálogo de los órganos de la provincia de Valladolid entre los años 1978 y 1980, trabajo que fue publicado por la Obra Cultural de la Caja de Ahorros Provincial de Valladolid en $1981 .{ }^{1}$

Una de las consecuencias de la realización de esta publicación derivó en la necesidad de fundar una asociación para la defensa y la protección del órgano castellano. Así, el 14 de agosto de 1980 se presentó la "Asociación Manuel Marín de amigos del órgano de Valladolid", agrupación que, tras su fundación, fue la abanderada del estudio, investigación y divulgación de la música de órgano, así como de la restauración de los instrumentos. ${ }^{3}$

Por aquel entonces, en 1982, la Sección Música de la Universidad de Valladolid estaba formada por $\mathrm{M}^{\mathrm{a}}$ Antonia Virgili Blanquet, lo que hacía inviable, o al menos muy complicado, asumir retos de investigación dentro del inmenso acervo documental sobre la historia de cada uno de los instrumentos de la provincia. Sin embargo, en 1992 comenzó a impartirse la Licenciatura en Historia y Ciencias de la Música, que se vio impulsada tras la adaptación al Grado en Historia y Ciencias de la Música y ampliada con el Máster de Investigación en Música Hispana posteriormente. Gracias a las palabras de la propia profesora Virgili, podemos acercarnos al conocimiento de algunas de las iniciativas que a lo largo de las tres últimas décadas se han tomado desde el ámbito universitario y a la Sección de Música que ella coordinaba, en el entorno del órgano histórico:

En estos años, desde la Universidad, con ayudas del Ministerio de Educación, de la Junta de Castilla y León, y de las Diputaciones se han impulsado trabajos diversos en relación al catálogo de órganos de la provincia de Valladolid, así como actuaciones en

Es importante recordar que José López Calo, en el número 524 de la revista Ritmo, definió este trabajo como "una obra que viene a cambiar conceptos fundamentales sobre lo que se sabía de los órganos históricos” (Cit. en Virgili 2005, p. 29)

2 Los socios fundadores fueron José Delfín del Val, Joaquín Díaz, Jesús Ángel de la Lama, Mariano Nieto, Pedro Aizpurúa, Manuel Fuentes, Lucía Riaño, María Fernández Calleja y María Antonia Virgili (Virgili, 2005, p. 16)

Todas las actividades realizadas por la Asociación están perfectamente reflejadas en el libro escrito por María Antonia Virgili que la propia Asociación publicó en 2005 para conmemorar su veinticinco aniversario. 
otras provincias de la autonomía. De Valladolid se realizó en la década de los noventa la informatización de todo el material publicado por Ángel de la Lama, actualizando el material gráfico e incorporando todas aquellas restauraciones o transformaciones que hubieran sufrido los instrumentos hasta aquel momento [...]. Gracias a las ayudas oficiales recibidas en las convocatorias de proyectos de investigación, en la actualidad contamos con los catálogos informatizados de las provincias de Burgos, León y Zamora, realizados al completo desde la Universidad [...], así como el de Ávila, Segovia y Soria, realizados por otros equipos, ajenos a la Universidad y a la asociación [Manuel Marín], pero de los que se realizó todo el proceso de informatización de los mismos. Sobre Palencia se defendió en la Universidad, en marzo de 1995, una tesis doctoral, realizada por la profesora Elena Le Barbier Ramos, cuyo objetivo primordial fue el estudio documental de la organería palentina. ${ }^{4}[\ldots]$ Los fondos documentales de la provincia de Zamora han sido trabajados por Virginia Flórez (2003), alumna de doctorado, quien defendió en 2003, en la Universidad de Valladolid, su trabajo de investigación para la obtención del Diploma de Estudios Avanzados con el título Estudio documental del patrimonio organístico de la diócesis de Zamora. (Virgili, 2005, pp. 30-33). ${ }^{5}$

\section{CONVENIOS INSTITUCIONALES DE COLABORACIÓN}

Es en este entorno en el que ya estaba consolidada una trayectoria de trabajos y equipos de investigación en el ámbito del órgano histórico dentro de la UVa, cuando el 29 de noviembre de 2002 se firma el "Convenio de colaboración entre la Excma. Diputación Provincial de Valladolid, la Universidad de Valladolid y el Arzobispado de Valladolid para la protección y difusión del órgano barroco en la provincia de Valladolid". ${ }^{6}$ Igualmente y con fecha un poco posterior - 27 de abril de 2004-, se firma el convenio con la Diputación Provincial de Palencia, el Arzobispado de Palencia y la propia Universidad de Valladolid, con objetivos idénticos, pero adscritos a los límites de su provincia.

En la documentación, todas las partes manifestaban una intención sinérgica hacia la "promoción, difusión, protección y restauración de los órganos barrocos como parte integrante del patrimonio histórico". Se iniciaba así una andadura de intervención integral (un "programa global", tal y como aparece reflejado en el texto) sobre estos instrumentos que a día de hoy continúa otorgando resultados sin faltar un ápice a la filosofía de fondo que lo puso en marcha.

El acuerdo se articula en cinco líneas maestras:

a. Conocimiento detallado de la situación de los órganos barrocos de la provincia de Valladolid y Palencia, para comprobar las necesidades de restauración.

b. Restauración de órganos barrocos seleccionados.

Esta tesis sería publicada por la Diputación de Palencia y la Institución Tello Téllez de Meneses (LeBarbier 2008).

Parte de este trabajo se encuentra disponible, aunque solo para los socios de Organaria, en http://www.organaria.es.

Aconsejamos visitar el enlace con la página de la Diputación en la que se recoge y difunde toda la labor realizada en estos años en el marco de la colaboración establecida entre la Universidad de Valladolid y dicha institución. http://www. diputaciondevalladolid.es/ciudadanos/ciu-cultura/organo_barroco/ 
c. Seguimiento de cada instrumento restaurado para conocer su necesidad de afinación, problemas de mecánica, etc.

d. Difusión del patrimonio musical relativo a los órganos barrocos mediante exposiciones didácticas y cursos específicos, propiciando con ello el conocimiento de estos instrumentos.

e. Fomento de la interpretación y audición de la música barroca para órgano, mediante actuación de uno o varios organistas con el fin de que se mantengan, suenen y recuperen su función.

Estas grandes líneas maestras se han concretado y resumido durante los años transcurridos en los siguientes cuatro aspectos:

a. Actividad de restauración de órganos barrocos.

b. Actividad de catalogación de órganos barrocos.

c. Uso de los órganos barrocos de la provincia.

d. Actividad de difusión del patrimonio organístico.

Aunque todas ellas están relacionadas con la salvaguarda y protección del Patrimonio organístico, ampliaremos brevemente cada una para centrarnos en la última, las actividades de difusión.

\section{ACTIVIDAD DE RESTAURACIÓN DE ÓRGANOS BARROCOS}

Se procedió a elaborar un listado de órganos susceptibles de restauración, para lo que se siguieron los siguientes criterios: interés artístico del instrumento, posibilidad de uso regular, interés local en la intervención y localización geográfica del mismo.

A fecha de junio de 2017, los órganos restaurados a través de este convenio de colaboración en la provincia de Valladolid, por orden cronológico, han sido los siguientes:

- Morales de Campos (Manuel Baquero, Gabino Ortega, 1791-1795). Restaurado por el taller de Ana Caramanzana y Berchtold Soergel, 2005.

- Torrelobatón (Fr. Cipriano Payueta OP. 1739). Restaurado por el taller de Carlos Álvarez, 2005.

- Villafrechós (Domingo Herbás, Pedro Antonio Ferreira, 1779). Restaurado por el taller de Luis Magaz, 2008.

- $\quad$ Tordesillas, iglesia de Santa María (Felipe Urarte, 1716). Restaurado por el taller de Joaquín Lois, 2011.

- $\quad$ Rueda (Francisco Ortega, 1747). Restaurado por el taller de Joaquín Lois, 2014.

- $\quad$ Nava del Rey, iglesia de los Santos Juanes (José de Alsúa, 1718). Restaurado por el taller de Joaquín Lois, 2015.

- Tiedra, ermita de Ntra. Sra de Tiedra Vieja (Francisco Henríquez, 1736). Restaurado por el Taller de Federico Acitores, 2016.

Cada una de las intervenciones anteriormente citadas ha sido objeto de un detallado seguimiento por parte de unas comisiones creadas a tal efecto y formadas por representantes 
de la propiedad (párrocos de los pueblos y el delegado de patrimonio del arzobispado), de la Universidad (profesores involucrados en el convenio), un técnico de la Diputación de Valladolid y, en la mayoría de los casos, se ha contado con expertos independientes para que emitieran un informe valorativo de la actuación sobre los instrumentos.

Puede parecer a priori que la actividad de restauración delimita en exceso la capacidad de difusión de resultados en relación con los contenidos patrimoniales. Para evitar esta "parcialización" de trabajos, dentro de cada restauración se exige a los organeros el diseño de un panel explicativo, colocado en lugar visible y redactado con un lenguaje apropiado, pero de fácil lectura, donde se reflejarán las características del mueble, los elementos propios de su sonoridad y parte de la historia del instrumento.

Figura 1. Panel explicativo de la restauración del órgano de Morales de Campos
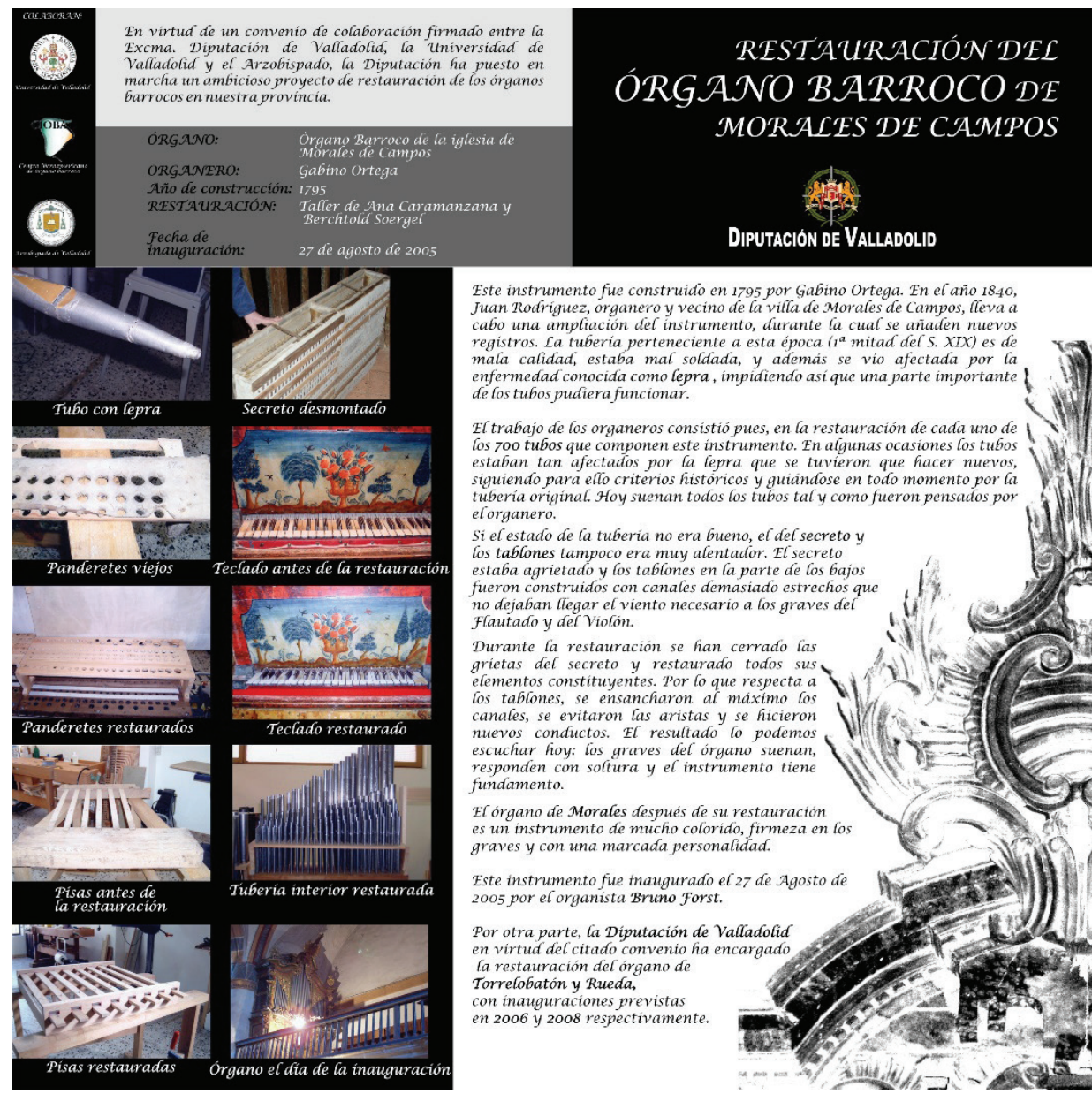

Este instrumento fue construido en 1795 por Gabino Ortega. En el año 1840, Juan Rodriguez, organero y vecino de fa vilfa de Morales de Campos, fleva a cabo una ampliación del instrumento, durante fa cual se añaden nuevos registros. La tuberia perteneciente a esta época ( $1^{a}$ mitad def $S$. XIX) es de mata cafidad, estaba mal soldada, y además se vio afectada por fa enfermedad conocida como lepra, impidiendo as que una parte importante de fos tubos pudiera funcionar.

El trabajo de los organeros consistió pues, en fa restauración de cada uno de fos 700 tubos que componen este instrumento. En afgunas ocasiones fos tufos estaban tan afectados por fa lepra que se tuvieron que facer nuevos. siguiendo para effo criterios historicos y guiándose en todo momento por fa tuberia original Hoy suenan todos fos tubos taf y como fueron pensados por tuberia origina

Si ef estafo fe fu tuberia no era bieno, ef fer secreto y Si el estado de la tuberia no era bueno, el del secreto tos tablones tampoco era muy alentador. Il secreto estaba agrietado y los tablones en la parte de los bajos fueron construidos con canales demastado estrechos que no dejaban llegar el vient Flautado y def Viofón.

Durante la restauración se han cerrado las grietas del secreto y restaurado todos sus efementos constituyentes. Por fo que respecta a tos tabfones, se ensancharon at maximo fos canales, se evitaron las aristas y se ficieron nuevos conductos. $\mathcal{E}$ resultado fo podemos escuchar hoy: los graves del órgano suenan, responden con softura y ef instrumento tiene fundamento.

Efórgano de Morafes después de su restauración es un instrumento de mucho colorido, firmeza en fos graves y con una marcada personafidad.

Este instrumento fue inaugurado ef 27 de Agosto de 2005 por el organista Bruno Forst.

Por otra parte, la Diputación de Valladolia en virtud del citado convento ha encargado fa restauracion del organo de Torrefobatón y Rueda, con inauguraciones previstas en 2006 y 2008 respectivamente.

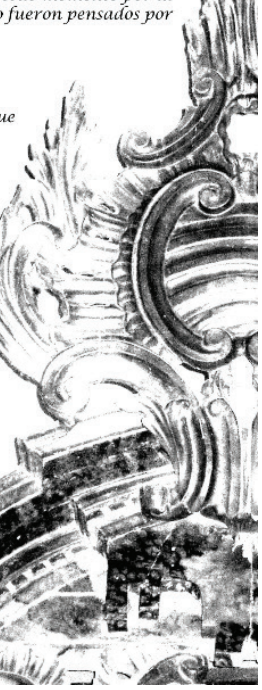




\section{ACTIVIDAD DE CATALOGACIÓN DE ÓRGANOS BARROCOS}

Como ya hemos adelantado en comentarios precedentes, hasta el momento se han desarrollado varios trabajos dentro de este epígrafe. Un paso previo a las actuaciones de restauración y difusión debe ser la obtención de un conocimiento detallado de la situación de los órganos de las provincias, para lo que se realizaron revisiones directas de todos los instrumentos existentes de cara a completar y actualizar los datos del catálogo original de Jesús Ángel de la Lama en el caso de Valladolid, y de los estudios comenzados por múltiples investigadores en la provincia de Palencia ${ }^{7}$. Se ha digitalizado toda la información y se ha procedido a la creación de un archivo gráfico completo de cada uno de los órganos. Además, a lo largo del proceso de actualización se localizaron varios instrumentos de los que no se tenía constancia hasta la fecha.

Esta línea de actuación ha obtenido su último resultado en la publicación en 2009, por parte de la Diputación de Valladolid, del libro Órganos Barrocos de la Provincia de Valladolid escrito por Ignacio Nieto Miguel, y en la publicación Órganos Barrocos de la Provincia y la Diócesis de Palencia (Hidalgo, Díaz-Emparanza \& Nieto, 2011).

Los restos y fragmentos sueltos de los órganos conservados en las distintas iglesias de la provincia de Valladolid fueron convenientemente almacenados y embalados en cajas para una mejor conservación.

Otra de las actividades relacionadas con la catalogación de órganos, si bien no reflejada desde el principio en los objetivos de los convenios, es el inventario de instrumentos históricos y fuentes musicales escritas, cuya primera fase ya ha tenido lugar y se espera poder ampliar las intervenciones en futuras ediciones. Es especialmente relevante la labor realizada en el Arciprestazgo de Campos, que alberga un variado y muy nutrido patrimonio artístico y cultural, como fruto de una intensa actividad humana desde los tiempos más remotos y de una fecunda situación económica, social y religiosa a lo largo de los siglos. El imponente patrimonio palentino es sumamente variado: importantes restos romanos (villas de la Olmeda o Quintanilla de la Cueza), visigóticos (San Juan de Baños de Cerrato, cripta de la catedral), así como un elevado número de manifestaciones románicas, góticas, renacentistas y barrocas, entre las que se sitúan algunas fundamentales y de referencia. Esta riqueza artística, histórica y cultural tiene un evidente eco en el campo que nos compete, el musicológico, pues de entre sus múltiples facetas y centrados en la organología musical, el aspecto quizá más sobresaliente y conocido es el de la organería.

Sin embargo, no conviene olvidar los dos problemas acuciantes que afectan a las provincias de Castilla y León. En primer lugar, la dispersión: el patrimonio se encuentra sumamente diseminado en localidades de escasa población. Ello ha supuesto y supondrá una dificultad añadida en la realización de las investigaciones, pues si nos fijamos en el caso de Palencia y tomamos como referencia la nada desdeñable cifra de 470 parroquias, hay que tener en cuenta que existe un número mucho mayor de edificios en los que se conserva este patrimonio musicológico: templos parroquiales, templos sufragáneos, ermitas, oratorios, seminarios, conventos y monasterios, museos, etc. En segundo lugar, la despoblación: la progresiva y preocupante situación demográfica supone un factor negativo, especialmente en los núcleos rurales, en los que no se produce el recambio generacional y existe una alta

De entre los múltiples autores destacamos Daniel Birouste y Sebastián de Castro, autores de La organería en Tierra de Campos (Birouste \& de Castro, 1979). 
tasa de envejecimiento, con lo que el patrimonio organístico corre el peligro de desgajarse de la comunidad que, en última instancia, le confiere sentido.

Si bien las operaciones de restauración han sido llevadas a cabo por maestros artesanos profesionales, todas estas actividades de catalogación fueron realizadas por estudiantes de Grado, alumnos de Doctorado y becarios de colaboración, adscritos por tanto a niveles educativos superiores, y supervisadas en todo momento por profesores de universidad. La actuación siempre tuvo como objeto principal el desarrollo de competencias vinculadas a la enseñanza del patrimonio histórico a través de aprendizajes de tipo constructivo y actividades dirigidas, todas ellas de valor académico constatado para el desarrollo de estudios posteriores (Fontal \& Ibáñez, 2015). La consolidación de conocimientos para que sirviera como recurso de aprendizaje sobre fuentes patrimoniales se ve así reforzada con actuaciones directas sobre el objeto de estudio.

\section{USOS DE LOS ÓRGANOS BARROCOS DE LA PROVINCIA}

Entre las intervenciones previas que facilitaron la inclusión de la actividad dirigida al mundo del órgano dentro de los convenios suscritos, destaca la serie de conciertos didácticos en Institutos de Enseñanza Secundaria de la provincia de Valladolid, que se desarrolló ininterrumpidamente durante un lustro y en los que participaron como intérpretes más de 12 grupos de distintos géneros musicales y cuyos beneficiarios fueron cerca de medio centenar de Institutos rurales de la provincia. El objetivo pedagógico era acercar músicas del mundo a un público joven mediante performances concertísticas pedagógicamente orientadas en las que se explicaban desde los instrumentos intervinientes hasta las formas musicales o los recursos sonoros que se presentaban a auditorios formados por estudiantes de educación secundaria con escasos o nulos conocimientos musicales previos.

Posteriormente, y vinculada a las actividades en torno al órgano histórico reflejadas en los convenios ya mencionados, surge la posibilidad de realizar dos tipos de intervenciones simultáneas: la programación de audiciones y el uso litúrgico de los instrumentos barrocos, ambas basadas en el principio de fomento de la audición e interpretación musical, pero contextualizada en el más complejo entramado de usos y funciones para los que, tanto los objetos sonoros como las composiciones ejecutadas, fueron concebidos. La puesta en valor de este tipo de patrimonio pasa por una doble vertiente, el valor del instrumento como bien inmueble por destino (Lefebvre, 2000) y el valor del patrimonio inmaterial que genera, es decir, la música misma. Desde un principio se planteó que el convenio de colaboración incluyera una vertiente sonora que se añade a la trayectoria con que la Diputación de Valladolid cuenta gracias a la organización del ciclo estival "El órgano y sus instrumentos", del que se han celebrado ya ocho ediciones consecutivas.

Surge así el ciclo denominado "Órgano en la Liturgia", en el que se trata de fomentar el uso del órgano en su contexto original: el oficio religioso. Es destacable señalar que, desde la firma del convenio, se han llevado a cabo más de 400 actuaciones en más de 25 localidades vallisoletanas con un alto grado de satisfacción por parte de los colectivos implicados: párrocos, comunidades parroquiales y ayuntamientos.

Este tipo de actuación, que excede al concepto del concierto ordinario en sentido estricto, resulta muy importante para la conservación de los propios instrumentos, pues responde a la intención de devolverles su función inicial, exclusivamente litúrgica, y al objetivo de que las personas de la localidad reasuman o adquieran un sentido de "propiedad" y de conciencia 
identitaria, herencia cultural o capital artístico muchas veces difuso. De alguna manera, se busca que se hagan responsables indirectos de su preservación, como agentes paralelos al propio bien inmueble, que comparten historia local y para que así lo valoren y lo defiendan en caso necesario o lleguen incluso a apoyar económicamente una posible restauración.

Los organistas participantes no son profesionales del instrumento, sin bien todos poseen conocimientos musicales y muchas veces un título de grado profesional de Conservatorio o equivalente en la especialidad, no se dedican profesionalmente a la interpretación. A menudo son estudiantes de alguna titulación vinculada con la música u organistas amateur adscritos a la población donde se efectúa el concierto.

\section{ACTIVIDADES DE DIFUSIÓN}

Esta línea de actuación sobre el patrimonio organístico de la provincia es una de las piezas medulares del convenio, ya que, con ella, se pretende transmitir y dar a conocer un acervo muchas veces minusvalorado o considerado ajeno, sobre todo entre los colectivos escolares, y conseguir con ello una puesta en valor de estos instrumentos y su consolidación como bien cultural de cada localidad. Para ello se han realizado diversos tipos de actividades:

\section{Realización de una exposición didáctica estable en el Centro Iberoamericano del órgano} barroco de Medina de Rioseco

En ella, a través de paneles explicativos, modelos tridimensionales, esquemas y otros apoyos gráficos, piezas, mecanismos y despieces de órganos, etc., se acerca a los estudiantes (tanto de enseñanza primaria como de secundaria) al mundo del órgano barroco. ${ }^{8}$ Desde su creación, en el año 2004, han pasado por la exposición más de cincuenta grupos de escolares de toda la provincia. ${ }^{9}$

Figura 2. Vista parcial de la exposición permanente sobre órgano barroco ubicada en Medina de Rioseco

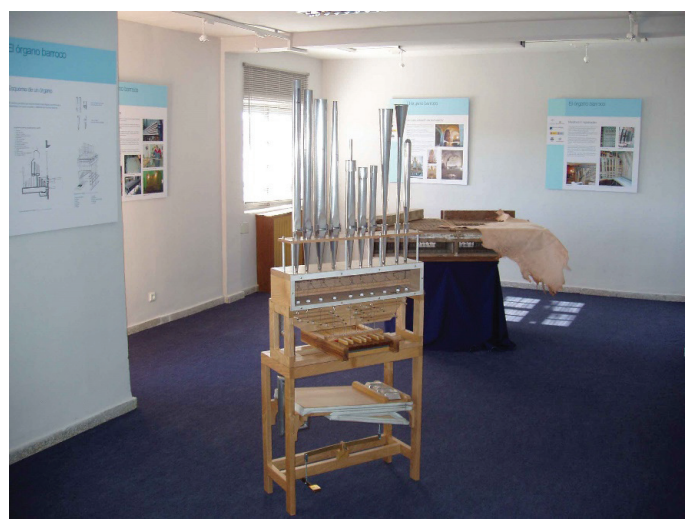

Es importante señalar que las actividades con escolares tienen una vertiente práctica, ya que tras la visita a la exposición se realiza una audición comentada en el órgano de Santa María de Mediavilla de la propia localidad (Díez \& Acitores, 1996).

9 A día de hoy la exposición está cerrada por problemas técnicos de infraestructura. 
En su planificación y diseño se elaboraron materiales didácticos adecuados a entornos educativos de enseñanza reglada, siempre en comunicación y colaboración con docentes de dichos centros, para adecuar y orientar el currículo a la idiosincrasia de los contenidos que se estaban desarrollando en ese momento. Tal es el caso de una maqueta de órgano diseñada ex profeso para la Universidad por el Taller de Organería Acitores en la que se desvela, a través de un panel de metacrilato y mediante elementos acondicionados de un órgano mecánico, la articulación de la mecánica interna de un órgano de tubos. Esta maqueta es transportable, permite la interacción - pues es necesario pisar un pedal para insuflar aire a los tubos y dispone de un teclado para hacer sonar los diez tubos disponibles-y resume de manera efectiva y visual el funcionamiento de un instrumento de este tipo.

Figura 3. Interior del díptico de las actividades didácticas anuales en el marco de la exposición permanente de Medina de Rioseco

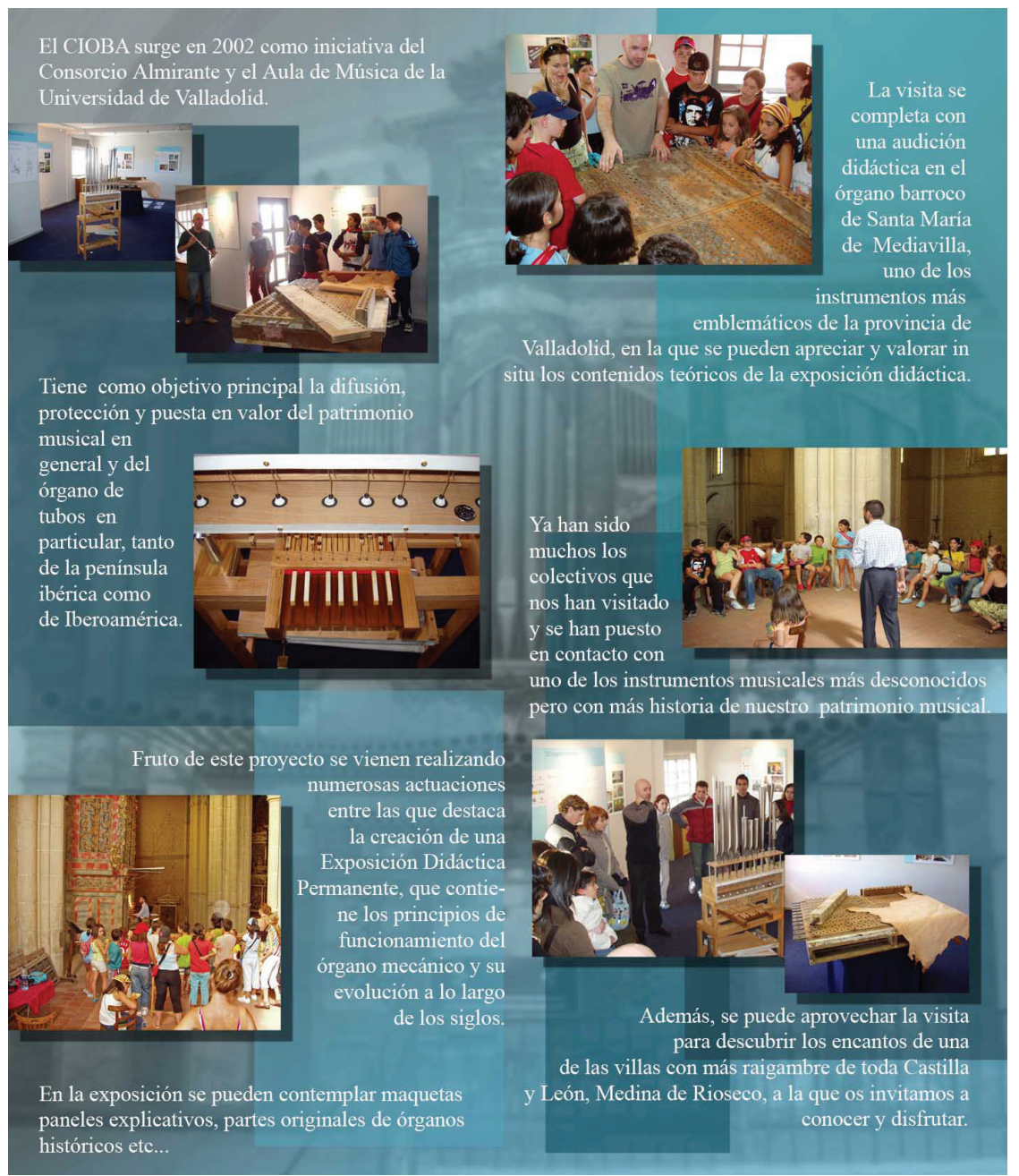


Igualmente, se diseñó un CD interactivo: "El órgano barroco: naturaleza e historia", cuyos contenidos versan sobre la vertiente didáctica de la historia del órgano Barroco en la Península Ibérica, dentro de un contexto general que abarca todos los instrumentos de este tipo, pero diferenciando los órganos barrocos por ciertas características propias que los identifican de entre el resto.

La disposición de los menús y los mismos contenidos están pensados para que sea usado por alumnos de Institutos de Enseñanza Secundaria y para que sirvan como material de apoyo curricular al profesorado. Material que fue facilitado a los profesores que visitaban la exposición para que lo utilizaran con otros grupos de alumnos y lo pudiesen integrar en las dinámicas habituales del proceso de enseñanza-aprendizaje. El mismo se distribuyó, posteriormente a todos los I.E.S. de Castilla y León, de cuyos docentes hemos recibido suficientes retroalimentaciones para poder concluir que los materiales les han resultado de mucha utilidad y que los han utilizado en numerosas ocasiones como complemento de información en sus programaciones anuales.

A modo de orientación, se presentan junto al $\mathrm{CD}$, pero dentro de una unidad didáctica suministrada a los docentes, los siguientes objetivos generales:

- Conocer las distintas manifestaciones musicales del órgano a través de la historia y su significación en el ámbito artístico y sociocultural.

- Desarrollar la capacidad de análisis de obras musicales como ejemplos de la creación artística; comprender su uso social y sus intenciones expresivas.

- Adquirir el vocabulario que permita explicar de forma oral y escrita los procesos musicales y establecer valoraciones propias.

- Iniciar la utilización de las nuevas tecnologías para la reproducción de la música y conocer su importancia en la creación de nuevas músicas.

El tema transversal más apropiado es vinculado a la Educación Moral y Cívica, definida como "respeto" y apreciación del instrumento.

Realización de conciertos didácticos para escolares en distintas localidades de la provincia y en la capital

A través de estos, dos profesores de la UVa se trasladan a los centros educativos y, en colaboración con las parroquias, realizan audiciones en vivo comentadas. Para los casos en los que estas actividades se efectúan en municipios en los que no se conserva órgano, se han preparado materiales didácticos específicos como presentaciones, cuestionarios, fragmentos o piezas de órgano cedidos por talleres de organería, etc., así como los materiales propios de la exposición permanente ya comentada. Hasta el momento se ha llevado a cabo una media de 10 actividades anuales con una valoración muy positiva por parte del profesorado y los alumnos, como así lo reflejan las cartas de agradecimiento dirigidas a la Diputación de Valladolid, entidad principal y patrocinadora de los convenios. 
Figura 4. Actividad didáctica en el órgano de la Iglesia de San Miguel de Peñafiel

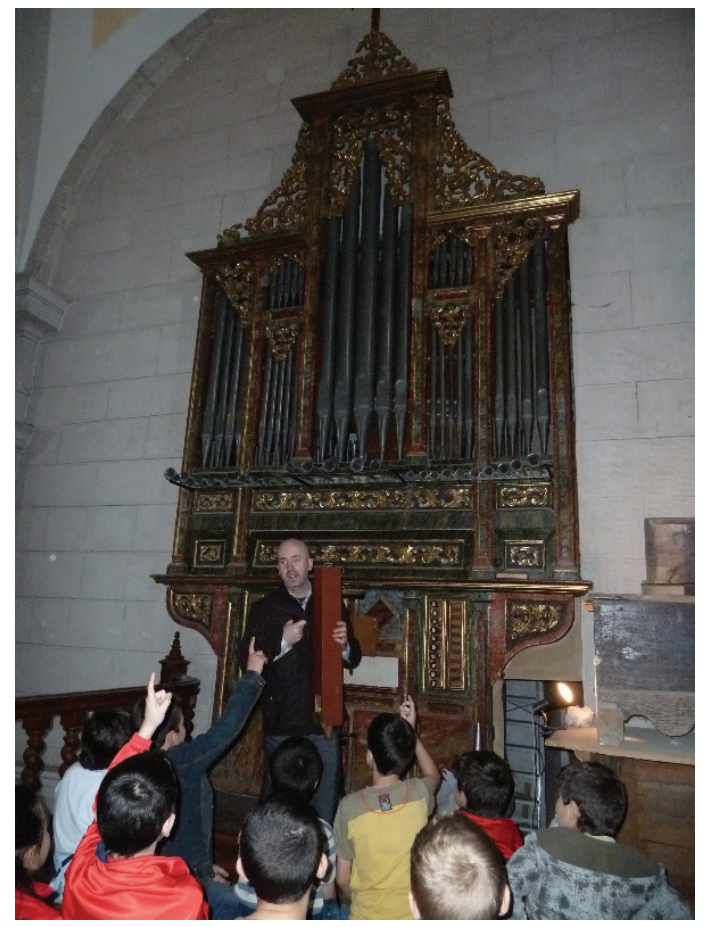

Material preparado con fines de difusión turística y promoción del patrimonio cultural

Especial resonancia y utilidad tiene, en este sentido, el material preparado con fines de difusión turística y promoción del patrimonio cultural relacionado con determinadas rutas publicitadas por la propia Diputación y en donde se ha insertado documentación sobre los órganos barrocos existentes en los recorridos e itinerarios seleccionados. Se dispone de una versión en tríptico informativo, así como una versión en archivo digital multiformato ejecutable bien desde un ordenador o bien desde dispositivos móviles.

\section{Elaboración de exposición itinerante en el Teatro Zorrilla de Valladolid}

En el año 2015 se organizó desde la Universidad de Valladolid una muestra sobre órganos barrocos que contenía importantes piezas y elementos mecánicos que resumían de una manera directa e inteligible la historia, el funcionamiento y los principios básicos de restauración de un instrumento antiguo. La exposición estuvo instalada y abierta al público general durante 4 semanas y obtuvo un gran impacto a tenor del éxito de asistencia. La ocasión fue aprovechada especialmente por docentes de institutos y colegios de la propia ciudad a través de un programa de visitas guiadas en que aprovecharon todos los materiales didácticos previamente elaborados y ensayados en anteriores ocasiones. 


\section{SUGERENCIAS DE ACTUACIÓN SOBRE EL PATRIMONIO. PROYECCIONES DE FUTURO}

Sobre la experiencia acumulada a raíz de todo lo anteriormente expuesto, consideramos que la dirección seguida en cuanto a la restauración de ejemplares históricos es la correcta, si bien cabe mejorar algunos aspectos como la pauta de actuación de las comisiones de seguimiento y la recepción de los instrumentos que sigue exigiendo todavía una mayor reflexión. Por lo demás, el balance resulta positivo y consideramos que es la actuación más sostenible (si en épocas de dificultades económicas la inversión en restauración baja, tan solo es cuestión de parcializar los trabajos y adaptarse a la disponibilidad de la institución), sistémica e interdisciplinar realizada en la provincia hasta el presente.

En lo que respecta a la catalogación de patrimonio musical, las insuficiencias detectadas son dramáticas: carecemos de un inventario de otros instrumentos musicales históricos de la provincia (posibles arpas o fragmentos de arpas barrocas, salterios, armonios, claves, pianos del siglo XIX y comienzos del XX, instrumentos de viento). Tampoco se ha realizado siquiera un trabajo de localización de fuentes audiovisuales que hayan podido conservarse: fonotecas de las radios, materiales en cintas abiertas u otros soportes, rollos de pianola, cilindros, discos de pizarra, discos de vinilo. Es una tarea pendiente y no solo del ámbito musical patrimonial. Por último, y en lo tocante a la dimensión documental de este mismo ámbito, el patrimonio musical de fuentes monódicas, e incluso de una polifonía primitiva, tanto en fragmentos sueltos como en posibles códices, permanece totalmente desconocido. Igual situación sufren los cantorales que consta se conservan en parroquias pequeñas y en otras bibliotecas, pero que todavía no se han podido catalogar en condiciones. Existen algunas excepciones relativas al patrimonio musical de fuentes polifónicas del XVI al XX, sobre el que se ha trabajado con rigor en los últimos años, ya que se han ido realizando inventarios de archivos y museos, pero urge la catalogación y digitalización de los materiales de audio existentes. Aun así, se trata de una mínima parte de lo que se conserva en la provincia y de todo aquello que no sabemos que se conserva. Por poner un único ejemplo: casi ninguno de los Monasterios de contemplativas, ni otros colegios de religiosos tienen inventariados sus fondos de música.

Sobre tales antecedentes, y siguiendo la vocación educativa desplegada alrededor del patrimonio que ha venido tratándose en las líneas anteriores, con poca inversión podrían plantearse programas anuales en el marco del convenio precitado para arbitrarse alguna dotación anual en forma de beca para alumnos dirigida a la catalogación de fondos musicales que, a la vez, tuviera carácter formativo. Igualmente, podrían utilizarse como medio de canalizar esfuerzos los trabajos prácticos del Máster de Música Hispana a la hora de organizar grupos de catalogación formados por los alumnos en prácticas y dirigidos por un tutor especializado. El artículo Educación patrimonial: retrospectiva y prospectivas para la próxima década, plantea estándares al respecto de los nuevos horizontes que se abren en educación patrimonial que se ajustan -con una muy acertada adecuación de los modelos educativos- a varias de las ideas aquí planteadas, con las que entra en consonancia (Fontal, 2016a).

En términos generales, consideramos que es fundamental difundir correctamente en medios de comunicación todas las actuaciones que se llevan a cabo con relación al patrimonio musical. La trasferencia de conocimiento se inicia en numerosas ocasiones a través de canales informales para centrarse más adelante en programaciones didácticas 
o diseños curriculares generalistas. No somos partidarios de multiplicar las actividades concertísticas, máxime dada la oferta existente en las ciudades y sus provincias, pero sí de apoyar acometidas dinamizadoras de localidades con menos oportunidades, así como iniciativas formativas acordes a la zona. Por supuesto consideramos que la labor educativa desarrollada durante estos años no debería detenerse: se demuestra eficaz, no supone grandes inversiones económicas y contribuye a la conservación y conocimiento del patrimonio. De hecho, se corresponde con parte de la secuencia procedimental lógica en la preservación del patrimonio que cita Olaia Fontal en varias fuentes de su autoría y que de manera rotunda resume en: “[...] conocer, comprender, respetar, valorar, sensibilizar, cuidar, disfrutar, transmitir" (Fontal, 2016b, p. 113).

Con relación al órgano barroco, consideramos que estaríamos ya en situación de plantear y diseñar la creación de un Centro de Interpretación del Órgano Barroco, único en España y que podría ser el eje de algunas de las actuaciones que hemos ido desarrollando en estos años y que han demostrado su eficacia, buen rendimiento y escaso coste.

\section{APORTACIONES DE LAS ACTIVIDADES FORMATIVAS}

Como paso previo a las conclusiones queremos mostrar en este apartado las principales contribuciones que ofrecen las actividades de formación, basadas en la taxonomía de enfoques y modelos de educación patrimonial identificados por $\mathrm{OEPE}^{10}$, ampliación a su vez de los modelos tradicionales de didáctica del Patrimonio (Fontal \& Marín, 2011), de los que extraeremos los más significativos y adecuados a las actividades descritas en este artículo.

a. Centrado en el docente. Los expertos que definen y transmiten los contenidos de las actividades utilizan métodos de enseñanza que atienden principalmente a cómo explicar el funcionamiento de un órgano a los distintos niveles educativos y tienen en cuenta qué competencias se deberían evaluar, aunque no sean los responsables directos de esta acción.

b. Centrado en el discente. Todos los contenidos se realizan teniendo en cuenta los conocimientos previos de los alumnos, siempre en comunicación previa con el docente del grupo que realiza la visita didáctica. Se trata de conseguir un grado razonable de aprendizaje significativo mediante metodologías activas. Se persigue que el alumno llegue a preciar su patrimonio y a valorar su propia realidad histórica (Fontal \& Marín, 2011).

c. Centrado en el contenido y en el contexto. Se trata del modelo principal, ya que los aspectos conceptuales y patrimoniales específicos constituyen el componente mayor de las actividades descritas, y se adaptan metodológicamente en función del aprendizaje y del medio. Por otro lado, los contenidos adquieren importancia en tanto el bien patrimonial que se pretende valorizar se halla en un entorno concreto, posee una identidad histórica y suele formar parte, en la mayoría de los casos, de un bien inmueble mayor, como una iglesia, catedral, ermita, o incluso un conservatorio de enseñanzas musicales regladas. Los materiales que describen la

10 Observatorio de Educación Patrimonial en España. 
realización de rutas turísticas de órganos entran de lleno en este modelo.

d. Centrado en el docente y en el discente. En este modelo las labores de docente se reparten entre el experto que ofrece unos contenidos en formato de visita didáctica -con experiencia pedagógica y amplio conocimiento del objeto patrimonial-y el docente de enseñanza formal. La labor de este último no se cuestiona, pero la inclusión de un segundo docente que adquiere el rol de docente no formal, si se nos permite la expresión adaptada al sujeto, le faculta para realizar una labor como mediador que, de facto, ya se considera respaldada, pues su labor se centra en estimular, despertar el interés y entusiasmar a través del elemento patrimonial.

Por otro lado, creemos que las actividades descritas poseen unas características pedagógicas concretas que las identifican en su integración con el bien patrimonial y que pasamos a enumerar:

- En relación con la aplicabilidad y transferencia, destacan por basar sus planteamientos metodológicos en los principios de preservación y promoción de patrimonio que promueve la UNESCO, son transferibles a otras áreas de conocimiento ajenas a la música, pero vinculadas al patrimonio y permiten su reorientación a entornos formales como el aula de música.

- En relación con el aprendizaje, las actividades de tipo formativo aquí presentadas mejoran significativamente el aprendizaje, permiten el acceso físico presencial a instrumentos históricos y desarrollan una justa valorización del patrimonio material e inmaterial del que el propio órgano es generador.

- Desde la óptica de la innovación, la creación de unos materiales digitales para los docentes y por extensión sus alumnos no es ninguna novedad, pero sí lo es la posibilidad de facilitar el, en ocasiones limitado, acceso a los instrumentos. La novedad reside también en la amplia recepción del público al que va dirigido, de distintos niveles educativos y provenientes de entornos formales, con la consiguiente mejora del tiempo invertido en el proceso y los mejores resultados tanto sobre el docente como el discente. Todo ello sin detrimento de la mejora directa en la preservación del patrimonio musical.

- $\quad$ Si ponemos la atención en las metodologías de aprendizaje, las visitas y conciertos didácticos son herramientas de enseñanza de contenidos tomadas desde perspectivas prácticas. Favorecen un entorno educativo inmersivo (la visita al propio instrumento en una iglesia o recinto que lo contenga) y se adecúan al nivel educativo al que se dirigen. Es labor del propio docente aplicar procesos de aprendizaje activos en relación con los materiales suministrados, a través de metodologías de trabajo guiado, cooperativo y sistemas de autoevaluación (en su caso).

- $\quad$ Si bien las TIC no son el mayor exponente de las actividades recogidas, los materiales digitales que se proporcionan al docente se establecen dentro del marco general de aplicabilidad de las TIC y suponen una herramienta más de las ya disponibles en ámbitos tradicionales (Mateos, 2010), tal y como destacan, y sobre las que inciden, todas las leyes educativas vigentes en España.

- Los ejemplos musicales y las unidades didácticas son recursos útiles de información; se asumen fácilmente en la programación de aula del docente y 
suponen un elemento de información al que de otra manera no tendrían acceso en su mayor parte. Pertenecen al tercer nivel de desarrollo de las nuevas tecnologías en educación (Asensio, Correa \& Grupo, 2011).

\section{CONCLUSIONES}

Son muchos los aspectos con los que podemos concluir tras lo expuesto, pero consideramos que dos son los principales. En primer lugar, la conveniencia de que las intervenciones sobre el patrimonio organístico sean planteadas de una manera integral. Y con esto nos referimos a que no solamente se intervenga en el bien físico, sino que se vaya más allá y se dé a conocer su resultado musical. ¿Qué sentido tiene restaurar si no se conoce el fin último para el que ese instrumento fue creado: sonar? Este planteamiento, si bien es mucho más difícil de llevar a cabo, hace sostenibles las políticas de restauración.

En segundo lugar, la pertinencia de que se firmen más convenios como el expuesto en este artículo. Un convenio tripartito que involucre no solo a la propiedad (la iglesia) y a la entidad financiadora (las diputaciones), sino que haya una tercera parte (la universidad, en este caso) que realice la necesaria intermediación entre las dos primeras y que sea la garantía de que la intervención en los instrumentos se haga con un criterio coherente y sostenible, para que este patrimonio lo puedan disfrutar y valorar en sus dimensiones física y sonora las generaciones venideras.

\section{REFERENCIAS BIBLIOGRÁFICAS}

Asensio, M., Correa, J., \& Grupo de Lazos de Luz Azul. (2011). Planteamiento inicial del proyecto Lazos de Luz Azul. Estándares de calidad en la utilización de la tecnología para el aprendizaje en museos y espacios de presentación del patrimonio. En M. Asensio, \& E. Asenjo (Eds.), Lazos de Luz Azul. Museos y Tecnologías 1, 2 y 3.0. (pp. 25-48). Barcelona: Editorial UOC.

Birouste, D., \& de Castro, S. (1979). La organería en Tierra de Campos. La obra de Tadeo Ortega. Palencia: Imprenta provincial.

Díez, Ma A., \& Acitores, F. (1996). El órgano de Santa María. Historia y Restauración. Valladolid: Junta de Castilla y León y Asociación "Manuel Marín" de Amigos del Órgano.

Flórez, V. (2003). Zamora: Documentación histórica del actual patrimonio organístico. Propuesta metodológica para su catalogación y estudio (Trabajo de Suficiencia Investigadora). Universidad de Valladolid, Valladolid.

Fontal, O. (2016a). El patrimonio a través de la educación artística en la etapa de primaria. Arte, Individuo y Sociedad, 28(1), 105-120.

Fontal, O. (2016b). Educación patrimonial: retrospectiva y prospectivas para la próxima década. Estudios Pedagógicos XLII,(2), 415-436.

Fontal, O., \& Ibáñez, A. (2015). Estrategias e instrumentos para la educación patrimonial en España. Educatio Siglo XXI, 33(1), 15-32. doi:10.6018/j/222481

Fontal, O., \& Marín, S. (2011). Enfoques y modelos de educación patrimonial en programas significativos OEPE. Educación artística Revista de investigación, 2, 91-96.

García, E. (1953). Documentos para el estudio del Arte en Castilla: maestros de hacer órganos. Anuario Musical, VIII, 210-229.

García, E. (1956). Documentos para el estudio del Arte en Castilla: maestros de hacer órganos. Anuario Musical, XI, 195-218. 
Hidalgo, B., Díaz-Emparanza, M., \& Nieto, I. (2011). Órganos Barrocos de la Provincia y de la Diócesis de Palencia. Palencia: Diputación de Palencia.

Lama, J. A. (1981). El órgano en Valladolid y su provincia: Catalogación y estudio. Valladolid: Obra Cultural de la Caja de Ahorros Provincial de Valladolid.

Le Barbier, E. (2008). La organería en la provincia de Palencia (1500-1800). Palencia: Diputación de Palencia e Institución Tello Téllez de Meneses.

Lefebvre, F. (2000). Memento práctico. Madrid: Lefevbre.

Mateos, S. (2010). Innovación en la comunicación global del patrimonio cultural: TIC. Patrimonio cultural de España, 4, 115-127.

Palacios, J. I. (Coord.). (2008). Órganos restaurados. Morales de Campos, Olmedo y Torrelobatón (Vol. 1). Valladolid: Diputación de Valladolid.

Virgili, M. A. (1981). La tradición organera vallisoletana en el siglo XX. Folklore, 5. Recuperado desde http://www.funjdiaz.net/folklore/07ficha.php?id=54

Virgili, M. A. (2005). El patrimonio organístico de Valladolid: veinticinco años de actuación. Asociación "Manuel Marín" de Amigos del órgano de Valladolid (1980-2005). Valladolid: Asociación "Manuel Marín" de Amigos del órgano de Valladolid. 